\title{
THE CONTINUOUS ITERATION OF REAL FUNCTIONS*
}

\author{
BY MORGAN WARD AND F. B. FULLER
}

1. Continuous Iterations. Let $E(x)$ be a real, continuous, steadily increasing function of $x$ in the range $-\infty<a \leqq x<\infty$ such that

$$
E(x)>x, \quad(x \geqq a),
$$

and let $E_{1}(x)=E(x), E_{2}(x)=E\left(E_{1}(x)\right), \cdots$ denote its successive iterates. In a previous note in this Bulletin, referred to hereafter as Note, one of us $\dagger$ has developed a simple formula for continuously iterating the function $E(x)$. We propose here to determine all continuous iterations of $E(x)$ subject to a restriction to be explained presently.

By a continuous iteration of $E(x)$ we shall understand a real function $\Theta_{y}(x)$ of the two real variables $x$ and $y$ with the following two properties

$$
\begin{aligned}
\Theta_{0}(x) & =x, \quad \Theta_{1}(x)=E(x), \quad(x \geqq a) . \\
\Theta_{y+z}(x) & =\Theta_{y}\left(\Theta_{z}(x)\right), \quad(x \geqq a, y, z \geqq 0) .
\end{aligned}
$$

The restriction which we shall impose upon the functions $\Theta_{\nu}(x)$ is the following:

(iii) $\Theta_{y}(a)$ is a steadily increasing continuous function of $y$ in the range $0 \leqq y \leqq 1$.

2. Prior Investigations. The continuous iteration of real functions was discussed in detail by A. A. Bennett. $\ddagger$ So far as the authors are aware, other investigators have confined their attention to the continuous iteration of analytic functions. $\S$ The functional equation (ii) was first considered by A. Korkine, $\|$ who

* Presented to the Society, February 29, 1936.

$\dagger$ Ward, Note on the iteration of functions of one variable, this Bulletin, vol. 40 (1934), pp. 688-690.

$\ddagger$ Annals of Mathematics, (2), vol. 17 (1916), pp. 23-69.

$\S$ See the references in the Note. 242 .

|| Bulletin des Sciences Mathématiques, (2), vol. 6 (1882), part 1, pp. 228 - 
proved formally a result equivalent to the first theorem of this paper, assuming that $\Theta_{y}(x)$ was differentiable with respect to $y$.

A complete discussion of the functional equation $E_{n}(x)=x$ with $x, E(x)$ real, $n$ a positive integer, has been given by $\mathrm{J}$. F. Ritt, ${ }^{*}$ and $W$. Chayoth $\dagger$ has recently proved certain very general existence theorems on functional equations in the real domain.

3. Theorem 1. Any function $\Theta$ satisfying the conditions (i), (ii), and (iii) is continuous and steadily increasing in both $x$ and $y$. Moreover for each such function $\Theta=E_{y}(x)$ there exists a unique, continuous, steadily increasing solution $\psi=f(x)$ of the functional equation

$$
\psi(x+1)=E(\psi(x)), \quad \psi(0)=0
$$

such that

$$
E_{y}(x)=f\left(f^{-1}(x)+y\right), \quad(x \geqq 0, y \geqq 0) .
$$

We have taken here and throughout the remainder of the paper, $a=0$ and $E(a)=1$ as was shown to be possible without loss of generality in the Note.

To prove this theorem, let $\Theta=E_{y}(x)$ be a particular function satisfying the conditions (i), (ii), and (iii). Since $E_{x+1}(0)$ $=E\left(E_{x}(0)\right)$, we see from (iii) that $E_{x}(0)$ is continuous and steadily increasing in the range $0 \leqq x<\infty$.

Write $f(x)$ for $E_{x}(0)$. Then $f(x)$ has a unique, continuous, steadily increasing inverse $f^{-1}(x)$ in the range $0 \leqq x<\infty$ such that

$$
f\left(f^{-1}(x)\right)=f^{-1}(f(x))=x, \quad f(0)=f^{-1}(0)=0 .
$$

Also $f(x+y)=E_{x+y}(0)=E_{y}\left(E_{x}(0)\right)=E_{y}(f(x))$. Hence $f(x+1)$ $=E(f(x))$, and $\psi=f(x)$ is a solution of (2). Then

$$
E_{y}(x)=E_{y}\left(f\left\{f^{-1}(x)\right\}\right)=f\left(f^{-1}(x)+y\right)
$$

* Annals of Mathematics, (2), vol. 17 (1916), pp. 113-122. See also the note by A. A. Bennett, loc. cit., p. 123.

$\dagger$ Monatshefte für Mathematik und Physik, vol. 39 (1932), pp. 279-288.

$\ddagger$ The converse of this theorem is well known. See, for example, A. A. Bennett, Annals of Mathematics, volume cited, pp. 74-75; pp. 23-30. 
which is (3). It is now evident that $E_{y}(x)$ is a continuous and steadily increasing function both of $x$ and of $y$.

Finally, the function $f(x)$ in formula (3) is uniquely determined by $E_{y}(x)$. For letting $x=y=0$, and using (1), we see that $f(0)=0$. Hence $f^{-1}(0)=0$. Therefore on letting $x=0$ and $y=x$, $f(x)=E_{x}(0)$. The problem of determining all continuous iterations of $E(x)$ is thus reduced to the solution of the functional equation (2).

4. THEOREM 2. Let $\theta(x)$ be a continuous function of $x$ in the interval $0 \leqq x<1$, which increases steadily from $\theta(0)=0$ to $\theta(1-0)=1$. Then every continuous steadily increasing solution $\psi$ of the functional equation (2) is of the form

$$
\psi(x)=E_{[x]}(\theta(x-[x])),
$$

where $[x]$ denotes the greatest integer in $x$.

Conversely, for every such choice of $\theta(x)$, (4) gives a continuous steadily increasing solution of the functional equation (2).

First of all, every such increasing solution $\psi$ of (2) tends to infinity with $x$. For assume that $\psi(x)$ tends to a finite limit $L$ as $x \rightarrow \infty$. Then $\psi(x)<L$ for all finite values of $x$. Now by (1), $E(L)>L$. Hence, since $E(x)$ is continuous, there exists a positive number $\delta$ such that $E(L-\delta)>L$. Choose $x_{0}$ so that $\psi(x)>L-\delta$, $x \geqq x_{0}$. Then $\psi(x+1)=E(\psi(x))>E(L-\delta)>L$, giving a contradiction.

It follows that in the interval $0 \leqq x<\alpha, \psi(x)$ has a unique, continuous, steadily increasing inverse $\phi=\phi(x)=\psi^{-1}(x)$ such that $\phi \rightarrow \infty$ as $x \rightarrow \infty$. This inverse is readily seen to satisfy the famous functional equation of Abel, ${ }^{*}$

$$
\phi(E(x))=\phi(x)+1, \quad \phi(0)=0 .
$$

For convenience, write $e_{n}$ for $E_{n}(0),(n=0,1,2, \cdots)$. Then $e_{0}=0, e_{1}=1$, and since by (1), $E(x)>x$, it follows that $e_{n}<e_{n+1}$.

We shall now show that $e_{n} \rightarrow \infty$. For otherwise, $e_{n}$ tends to a finite limit $k$, and $e_{n}<k,(n=0,1,2, \cdots)$. Since $k>1$, if $E_{-1}(x)$ denotes the inverse of $E(x)$, then $E_{-1}(k)=M$, where $0<M<k$. For $k=E\left(E_{-1}(k)\right)=E(M)>M$. Hence for all sufficiently large $n$, $e_{n}>M$. But then $e_{n+1}=E\left(e_{n}\right)>E(M)=k$, giving a contradiction.

* Works, vol. 2, Posthumous Papers, 1881, pp. 36-39. 
It follows that, given any positive value of $x$, we can determine an integer $k$ such that

$$
e_{k} \leqq x<e_{k+1} .
$$

Let $x$ lie in the interval (6). Then from the properties of $E(x)$ and its ordinary iterates, we can write

$$
x=E_{k}(y), \quad y=E_{-k}(x), \quad(0 \leqq y<1),
$$

where $E_{-k}(x)$ denotes the inverse of $E_{k}(x)$ in the interval $e_{k} \leqq x<\infty$.

Now in the interval $0 \leqq x<1$, let us write $\theta^{-1}(x)$ for $\phi(x)$. Then $\theta^{-1}(0)=0$, and $\theta^{-1}(x)$ increases steadily and continuously as $x$ increases, and $\theta^{-1}(1-0)=\lim _{x \rightarrow 1} \theta^{-1}(x)=1$ by (5). Furthermore, the inverse of $\theta^{-1}(x)$, which we denote by $\theta(x)$, exists and has the properties stated in Theorem 2. From (5), we see that

or

$$
\phi(x)=\phi\left(E_{k}(y)\right)=\phi(y)+k=\theta^{-1}(y)+k
$$

$$
\phi(x)=\theta^{-1}\left(E_{-k}(x)\right)+k .
$$

Since $0 \leqq E_{-k}(x)<1$, we observe also that $k=[\phi]$, the greatest integer in $\phi(x)$.

To determine $\psi$, we need only solve (7) for $x$ in terms of $\phi$. We have

Hence*

$$
\begin{aligned}
\phi-[\phi] & =\theta^{-1}\left(E_{-[\phi]}(x)\right), \\
\theta(\phi-[\phi]) & =E_{-[\phi]}(x), \\
x & =E_{[\phi]}(\theta(\phi-[\phi])) .
\end{aligned}
$$

$$
\psi(x)=E_{[x]}(\theta(x-[x])) .
$$

The proof of the converse for a function $\theta$ satisfying the conditions of the theorem is almost word for word the same as in the special case $\theta(x)=x$, which has been given in full in the Note.

The function $\theta(x)$ is arbitrary save for the restrictions stated in the theorem. Once chosen, it fixes the iteration completely; it is in fact $E_{x}(0)$.

California Institute of Technology and Whittier, Calif.

* It is obvious that if $\pi(x)$ denotes a periodic function of $x$ with period one, such that $\pi(x)=\theta(x),(0 \leqq x<1)$, then we can write $\psi(x)=E_{[x]}(\pi(x))$, or more concisely still, $\psi(x)=E_{[x]}(\psi(x-[x]))$, since $\psi(x-[x])=\theta(x-[x])$. 